\title{
Intersecciones de identidad de género, personas trans, migración, trabajo sexual y su relación con la trata de seres humanos
}

\author{
Sebastián Maldonado Vivar ${ }^{1}$ y Samuel Erices Riquelme ${ }^{2}$
}

\section{RESUMEN}

El presente artículo aborda la relevancia de considerar las intersecciones de identidad de género, personas trans, migración, trabajo sexual y su relación con la trata de seres humanos, a través de la experiencia de trabajo de campo realizado entre 2015 y 2018 con el Sindicato chileno de trabajadoras sexuales Trans "Amanda Jofré Cerda".

Se pretende visibilizar este fenómeno poco abordado, a partir de los hallazgos evidenciados en el trabajo de campo. Su desarrollo teórico expone las dicotomías del marco normativo chileno, la ausencia del enfoque de derechos humanos, la crítica Constitución, que no representa la protección de los mismos, y la necesidad de las personas trans de asociarse en espacios comunes desde la perspectiva de la asociatividad, vinculada al fenómeno de la trata de seres humanos. El artículo también examina la invisibilización de las víctimas transexuales, destacando la interseccionalidad como un enfoque aplicado al abordaje de problemáticas sociales vinculadas con los derechos humanos, y visibilizando a las víctimas de trata trans, cuyo delito se oculta al tratarse de personas que, históricamente, no han sido reconocidas como una identidad de género dentro de las culturas y países patriarcales de América del Sur.

El escrito finaliza con una serie de conclusiones contemporáneas que subrayan las necesidades de las víctimas de trata trans, las cuales exhiben una fuerte relación entre la interseccionalidad, las relaciones de poder y la vulneración de derechos a la que se encuentran expuestas.

1 Trabajador Social, Magíster en Salud Pública, Director de COSAM La Pintana, Académico Escuela de Derecho y Humanidades, carrera de Trabajo Social Facultad de Derecho y Humanidades, Universidad Central de Chile, E-mail: tsmaldonadov@gmail.com

2 Trabajador Social, Magíster en Intervención Social, Académico y Secretario de Estudios de la Escuela de Derecho y Humanidades, carrera de Trabajo Social Facultad de Derecho y Humanidades Universidad Central de Chile, E-mail: samuel.erices@ ucentral.cl 
Intersecciones de identidad de género, personas trans, migración, trabajo sexual y su relación con la trata de seres humanos /Maldonado y Erices

Palabras clave: trabajo sexual, personas trans, trata de seres humanos, asociatividad, interseccionalidad

\title{
Intersections of Gender Identity, Transgender People, Migration, Sex Work and Their Connection to Sex Trafficking
}

\author{
ABSTRACT
}

This article addresses the importance of considering the intersections of gender identity, transgender people, migration, sex work and their connection to sex trafficking, through the fieldwork experience performed between 2015 and 2018 with the Chilean Transgender Sex Workers Union "Amanda Jofré Cerda."

The article looks to raise awareness of this little-known phenomenon, based on the findings generated in the fieldwork. Its theoretical development reveals the dichotomies of the Chilean regulatory framework, the absence of human rights approach, criticism of the Constitution which does not protect these rights, and transgender people's need to come together in common spaces from the perspective of associativity, tied to the phenomenon of sex trafficking. The article also examines the invisibilization of transsexual victims, highlighting intersectionality as an approach that can be applied to address social issues related to human rights and to draw attention to the transsexual victims of sex trafficking, often forgotten because they have historically not been recognized as a gender within the patriarchal cultures and countries of South America.

The paper ends with a series of contemporary conclusions that highlight the needs of transgender sex trafficking victims, which are closely related to intersectionality, power relations and the violation of their rights.

Key words: sex work, transgender people, sex trafficking, associativity, intersectionality

\section{Intersecções de identidade de gênero, pessoas trans, migração, trabalho sexual e sua relação com o tráfico de seres humanos}

\section{RESUMO}

O presente artigo aborda a relevância de considerar as intersecções de identidade de gênero, pessoas trans, migração, trabalho sexual e sua relação com o tráfico de seres humanos, através da experiência de trabalho de campo realizado entre 2015 e 2018 com o Sindicato Chileno de Trabalhadoras Sexuais Trans "Amanda Jofré Cerda”.

Pretende-se visibilizar este fenômeno pouco abordado, a partir dos descobrimentos evidenciados no trabalho de campo. Seu desenvolvimento teórico expõe as dicotomias do sistema normativo chileno, a ausência do 
enfoque de direitos humanos, a crítica Constituição, que não representa a proteção dos mesmos e a necessidade das pessoas trans de se associarem em espaços comuns desde a perspectiva da associatividade, vinculada ao fenômeno do tráfico de seres humanos. O artigo também examina a invisibilização das vítimas transexuais, destacando a interseccionalidade como um enfoque aplicado à abordagem de problemáticas sociais vinculadas com os direitos humanos, e visibilizando as vítimas do tráfico trans, cujo delito é oculto ao se tratarem de pessoas que, historicamente, não foram reconhecidas como uma identidade de gênero dentro das culturas e países patriarcais da América do Sul.

O artigo finaliza com uma série de conclusões contemporâneas que destacam as necessidades das vítimas do tráfico trans, as quais exibem uma forte relação entre a interseccionalidade, as relações de poder e a vulneração dos direitos a que estão expostas.

Palavras-chave: trabalho sexual, pessoas trans, tráfico de seres humanos, associatividade, interseccionalidade

\section{Introducción}

En la historia sociojurídica contemporánea, los marcos normativos van configurando realidades sociales que, en rigor, deberían responder a la bien llamada "cuestión social". En consecuencia, las violaciones a los derechos humanos se configuran en escenarios donde los marcos legales y constitucionales son favorables para ciertos grupos privilegiados (en su mayoría económicos), dejando excluidos a grupos específicos y poco visibilizados, como son las personas trans. Por ello, en este escrito se abordarán conceptos relacionados con concepciones que predominan ante el trabajo sexual, la trata de personas y las personas trans.

De acuerdo con la Biblioteca del Congreso Nacional de Chile ${ }^{3}$, se plantea la existencia de dos discursos en relación al "trabajo sexual": uno relacionado con la voluntad y elección, por lo tanto, con derechos y deberes y, en consecuencia, debe ser considerado un trabajo, y otra visión determinista que explica la prostitución como una salida inexorable a variados acontecimientos económi-

3 La regulación de la prostitución en la legislación comparada. BCN, 2005. Recuperado en junio de 2014 de http://www.bcn.cl/bibliodigital/pbcn/estudios/estudios_pdf_ estudios/nro325.pdf 
cos, socioculturales, sexuales y psicológicos, considerándolo como una de las formas de violencia más extrema en contra de la mujer.

Respecto de la trata de seres humanos, conocida como trata de personas en Chile, la Ley No 20.507 de 2011 introdujo modificaciones en el Código Penal, definiendo los delitos del tráfico de migrante y de trata de personas, estableciendo penas y multas, $y$ considerando como agravante que las víctimas sean menores de $e^{\text {edad }}{ }^{4}$. El delito de "trata de personas" está tipificado en el artículo 411 quáter del Código Penal:

El que, mediante violencia, intimidación, coacción, engaño, abuso de poder, aprovechamiento de una situación de vulnerabilidad o de dependencia de la víctima, o la concesión o recepción de pagos u otros beneficios para obtener el consentimiento de una persona que tenga, acoja o reciba personas para que sean objeto de alguna forma de explotación sexual, autoridad sobre otra, capte, traslade, incluyendo la pornografía, trabajos o servicios forzados, servidumbre o esclavitud o prácticas análogas a ésta, o extracción de órganos, será castigado con la pena de reclusión mayor en sus grados mínimo a medio y multa de cincuenta a cien unidades tributarias mensuales. Si la víctima fuere menor de edad, aun cuando no concurriere violencia, intimidación, coacción, engaño, abuso de poder, aprovechamiento de una situación de vulnerabilidad o de dependencia de la víctima, o la concesión o recepción de ésta.

Además, cabe destacar que se debe diferenciar entre trata de personas con fines de explotación y trabajo sexual. Ante ello, los acuerdos internacionales entregan la tipificación de delito a la explotación sexual, sancionado a través del Protocolo para prevenir, reprimir y sancionar la trata de personas, especialmente de mujeres y niños, que complementa la Convención de las Naciones

4 Recuperado de https://www.leychile.cl/Navegar?idNorma=1024319 Consultado 01/12/2019 20:36 
Unidas contra la delincuencia organizada transnacional. En Chile, a través de la Subsecretaria de Prevención del Delito, se estableció un Protocolo intersectorial de atención de víctimas de trata de personas.

Referente al trabajo sexual, no existen acuerdos internacionales. La $\mathrm{OMS}^{5}$ lo define como sexo consentido voluntario entre adultos a cambio de dinero o bienes. La coalición contra el tráfico de mujeres y la Red Internacional de Derechos Humanos postula que todo trabajo sexual, independiente de que exista o no intercambio de dinero o bienes, lleva implícita cierta explotación.

En la base de lo que se entiende por comercio sexual, encontramos enfoques como el prohibicionista, que afirma que todo comercio sexual es un delito (Irlanda); el abolicionista, que señala que la prostitución es una forma de esclavizar a las personas (Suecia); el reglamentarista, que presenta ciertas excepciones en el Código Penal para el trabajo sexual que cumple con ciertas condiciones, y el laboralista, que lo equipara con los derechos de cualquier otra profesión formalmente reconocida (Alemania).

Respecto de los derechos de identidad de las personas trans, la Ley 21.120 reconoce y da protección a la identidad de género, $\mathrm{cu}-$ ya ley recién fue publicada en el Diario Oficial el 10 de diciembre de 2018, reconociendo así la facultad de toda persona, cuya identidad de género no coincida con su sexo y nombre registral, de solicitar la rectificación de estos ${ }^{6}$.

\section{Metodología}

El trabajo es de enfoque cualitativo mediante entrevistas semiestructuradas, desde el enfoque fenomenológico y dialéctico. Asimismo, el diseño es descriptivo observacional participante, usan-

5 Organización Mundial de la Salud

6 CF:https://www.bcn.cl/leyfacil/recurso/ley-de-identidad-de-genero consultado: 0312-2019 20:51 
do apuntes de trabajo de campo, considerando la revisión de fuentes bibliográficas que dan soporte a lo expuesto.

Se entrevistó a 20 mujeres trans durante los años 2015-2018 pertenecientes al Sindicato chileno de trabajadoras sexuales Trans Amanda Jofré Cerda.

\section{Antecedentes de la situación en Chile}

La "prostitución", entendida como "el intercambio de comportamientos de base sexual a cambio de un bien o servicio valorado por la contraparte"7, es un fenómeno complejo, diverso y con una multiplicidad de factores que intervienen en él: las migraciones, feminización de la pobreza y de la subsistencia, falta de acceso a derechos sociales y culturales, consumismo generalizado, las personas mismas en situación de prostitución — generalmente mujeres-, las organizaciones que lucran con la prostitución y quienes consumen prostitución, mayoritariamente varones (Zaldúa, Longo y Sopransi, 2007). Lo descrito entrega aportes para comprender las dinámicas del comercio sexual. Cabe señalar que no existe una reglamentación que permita un comercio sexual justo, organizado y protegido en los países de América del Sur. La obtención de cifras confiables relacionadas con el trabajo sexual es tarea difícil pues, independientemente del enfoque legal usado por los diferentes países, estos presentan dificultades para dar cuenta de la temática. Respecto del fenómeno de la trata de personas, la Organización Internacional del Trabajo (OIT), en su documento "Estimación Mundial sobre el Trabajo Forzoso", estima que el 22\% (4.500.000 personas) de las personas traficadas son víctimas de explotación sexual forzada y, de éstas, la mayoría son mujeres y niñas. Asimismo, según el Informe Mundial sobre la Trata de Personas, 2012, de la Oficina de las Naciones Unidas contra la Droga

7 La regulación de la prostitución en la legislación comparada. BCN, 2005. Recuperado en junio de 2014 de http://www.bcn.cl/bibliodigital/pbcn/estudios/estudios_pdf_ estudios/nro325.pdf 
y el Delito (UNODC), correspondería al 58\% de todo el tráfico de personas detectado en todo el mundo ${ }^{8}$.

Por otro lado, corrientes feministas críticas han instalado el comercio sexual - desde ahora trabajo sexual - como una forma de dominación del sistema patriarcal, lo que hace mucho más complejo legislar en la materia, pues se cruzan cuestiones éticas, históricas, morales, legales, entre otras.

En el caso de Chile, el trabajo sexual no es tipificado como delito, siempre y cuando sea cometido bajo la responsabilidad y consentimiento propio con fines individuales. La dimensión reglamentarista del trabajo sexual en Chile ha estado explicitada a partir del código sanitario y del reglamento de enfermedades de transmisión sexual (Santander, Primera y Barrera, 2009), por lo que el Estado de Chile, a través del Ministerio de Salud, tiene la obligación de regular la actividad mediante el reglamento mencionado, con el fin de controlar la propagación de enfermedades sexuales transmisibles, incluyendo la indicación de que las mujeres que lo ejerzan deberán portar un carnet acreditando su salud sexual (Norma, Medidas y La, 2013).

El año 2012 el Ministerio del Interior y Seguridad Pública realizó el primer diagnóstico de trata de personas, en el que se evidenció, entre 2007 y 2011, ciento trece denuncias relacionadas con la trata, lo que equivale a un promedio de 23 denuncias por año (Departamento de Crimen Organizado, División de Estudios, 2012).

Por su parte, el estudio de trata de la Organización Internacional para las Migraciones (OIM), realizado en 2008 para Chile, reporta la presencia de 36 casos y 147 víctimas asociadas. Dicho estudio revela que los casos corresponden en un $18 \%$ a niños y niñas, y en un 58\% a mujeres adultas (IOM/Chile, 2008). En relación con los fines del tráfico de personas, el $50 \%$ de las víctimas fue con fines

8 La regulación de la prostitución en la legislación comparada. BCN.2005. Disponible en: http://www.bcn.cl/bibliodigital/pbcn/estudios/estudios_pdf_estudios/nro325. pdf (Junio, 2014) 
de explotación sexual, el 40\% de explotación laboral, mientras que el 10\% se encontraba en tránsito (Ibíd).

Si bien la realidad mundial es categórica al definir el delito de trata de personas, incluyendo los modelos explicativos de tipificación del fenómeno (trata, tráfico y explotación), entre otros, en el ámbito de caracterización global del fenómeno se carece de información profunda respecto de las víctimas, pues no existen datos de las personas trans que son víctimas del delito de trata, solapando esta realidad en burdeles, cafés, bares y comercio sexual obligado, sin desconocer que esto afecta de manera transversal a las personas, incluidas las no trans; no obstante, una de las características que más se repiten en la vivencia de las personas trans, en este tipo de explotación sexual, es justamente la obligatoriedad en ejercerlo, la coacción y, por sobre todo, con dos relatos: para algunas un elemento de subsistencia, para otras un elemento de coacción.

\section{Ser migrante, transexual y trabajadora sexual en Chile}

Primero que todo, se hace muy complejo caracterizar el fenómeno, al tratarse de que, en América del Sur, existen pocos estudios al respecto. Citando un estudio descriptivo de salud de migrantes en España (2014) - para poder caracterizar el fenómeno-, se señaló que: "Los hombres latinos transexuales presentan una alta prevalencia de VIH y sífilis" (Delgado-Rodríguez, 2014). Más lapidario es aún el estudio argentino de "Exigibilidad de derechos de personas en situación de prostitución y dispositivos comunitarios en CABA", en el que existe una relación entre la esperanza de vida y la condición de personas travestis y transexuales, la cual oscila entre los 38 años. (Zaldúa, Longo y Sopransi, 2007).

Por lo que podríamos indicar que existe un elemento basal y muy desventajoso respecto de otros grupos específicos de la sociedad: la esperanza de vida, bien ejemplificada en el estudio argentino, así como también la alta presencia de trans masculinos; conjetu- 
rando que, eventualmente, ser trans en contextos desfavorables ya configura una significativa baja en la esperanza de vida, un elemento más para fundamentar la vulnerabilidad profunda de este grupo específico viviendo en Chile.

El Sindicato chileno de trabajadoras sexuales Trans Amanda Jofré Cerda nació hace 17 años, adoptando el nombre de la injustamente asesinada Amanda (Rodolfo) Jofré Cerda (dirigente social icónica del mundo trans en aquellos años). Este fue el lugar donde se realizó el trabajo de campo.

Actualmente, el Sindicato agrupa a más de 200 socias, chilenas y extranjeras, aproximadamente unas 80 activas, que desarrollan el trabajo sexual en Santiago y otras regiones, y cuya instancia tiene representación nacional. Los objetivos del sindicato son representar a las socias, visibilizar el trabajo sexual, relevarlo en dimensiones legales y de protección desde el Estado, como también colaborar con abogacía política, asistencia social, vinculación sanitaria, representación legales y documentación de casos de asesinatos y homicidios de personas trans, entre otras formas de violencia hacia éste grupo de personas. En esta línea, el trabajo sexual vinculado a la trata de seres humanos y personas trans migrantes configura un fenómeno social que no es constitutivo de garantías mínimas legales (desde la legalización de servicios sexuales hasta las mismas leyes de identidades de géneros), estableciendo vacíos legales que dejan en absoluto abandono y vulneración a grupos específicos como los que aquí se describen.

\section{La trata de personas o trata de seres humanos}

Un elemento interseccional, delictual y criminal en el ámbito de los derechos humanos es el análisis de la "trata de personas" - o "trata de seres humanos"-. Este es un fenómeno social complejo, transregional. En el mundo existen 27 millones de esclavos modernos (Datta \& Bales, 2013) que podrían ser parte de este delito. La compraventa de seres humanos constituye un mercado lucrativo, que moviliza más de 32 mil millones de dólares al año, afec- 
tando a 161 Estados y a un estimado de 12,3 millones de personas víctimas al año, a nivel mundial (UNODC, 2014).

En todo el mundo, las víctimas de 152 nacionalidades diferentes han sido identificadas en 124 países; se ha detectado al menos 510 flujos de tráfico de personas; cerca del 64\% de los traficantes condenados son ciudadanos del país de condena; aproximadamente el $72 \%$ de los traficantes condenados son hombres y el 28 por ciento mujeres; un $49 \%$ de las víctimas detectadas son mujeres adultas, mientras que un $33 \%$ de las víctimas son niños, lo cual es un aumento del 5\% en comparación con el informe 2007-2010 (UNODC, 2014).

Si bien las diferencias regionales son notorias respecto del fin último de la trata de personas, en el caso de Europa y Asia Central la trata es con fines de explotación sexual; en Asia Oriental y el Pacífico es la explotación con fines de trabajo forzoso; en el caso de América los porcentajes son idénticos en relación con ambos tipos de trata. La relación en América, respecto de adultos y niños tratados, equivale a un $31 \%$ niños y un $69 \%$ adultos (UNODC, 2014).

El cruce interseccional entre las relaciones de poder, también solapadas en la caracterización de las víctimas de trata trans, es parte de la cultura patriarcal dominante y opresora. Como plantea Viveros:

Desde hace algunos años, la interseccionalidad ${ }^{9}$ se ha convertido en la expresión utilizada para designar la perspectiva teórica y metodológica que busca dar cuenta de la percepción cruzada o imbricada de las relaciones de poder. Este enfoque no es novedoso dentro del feminismo y, de hecho, actualmente existe un acuerdo para señalar que las teorías feministas habían abordado el problema antes de darle un nombre. (Viveros Vigoya, 2016)

9 El concepto mismo de interseccionalidad fue acuñado en 1989 por la abogada afroestadounidense Kimberlé Crenshaw, en el marco de la discusión de un caso concreto legal, con el objetivo de hacer evidente la invisibilidad jurídica de las múltiples dimensiones de opresión experimentadas por las trabajadoras negras de la compañía estadounidense General Motors. 


\section{Conclusiones}

\section{En el trabajo de campo}

Los factores protectores son muy escasos en las personas trans, frente a diversas dimensiones, como por ejemplo "ser migrante, transexual y trabajadora del comercio sexual" es una de las situaciones más desfavorables, viéndose envueltas en complejas y altas vulnerabilidades, entre ellas la trata de seres humanos, el tráfico de personas, la explotación sexual y trabajos forzados, los proxenetismos, entre otra serie de factores de riesgo a los que se ven expuestas. Además, el trabajo de campo contempló dimensiones en salud, educación, registro civil, acceso a la justicia, trabajo, derecho a la identidad.

\section{Los elementos sororos del sindicato}

Cuando leemos la palabra "sororidad" creemos, a simple vista, confundirla con el conocido concepto "solidaridad", cosa no casual en un mundo moderno que instala nuevas conceptualizaciones sin realizar un profundo análisis de cómo las sociedades se van configurando desde el lenguaje y lo verbal.

Sororidad es, en lo concreto, "un pacto", una alianza entre mujeres, y no necesariamente mujeres biológicas propiamente tales. Acuñando las reflexiones de Lagarde de los Ríos,

no es una casualidad que las mujeres hagan pactos cuando se encuentran en lo público y ahí están el lenguaje y las maneras de relacionarse para lograr el acuerdo de intervenir, proponer, impulsar o ejecutar tantas cosas. Pacto, agenda y ciudadanía van de la mano. Tienen sus antecedentes en el apoyo solidario directo, privado, tantas veces clandestino y subversivo entre una y otra. (Lagarde y de los Ríos, 2009)

Lagarde insiste en que el pacto conlleva un sin fin de situaciones sintomáticas de la historia. Es preciso reconocer que la cultura femenina tradicional vigente no incluye conocimientos, habilidades 
y destrezas para agendar ni pactar; que muchas aprenden en el estilo masculino y patriarcal para luego desaprenderlo, al sentir cuán contradictorio es que se conduzcan así entre ellas, lo estéril de ese proceder y la necesidad de construir la alianza entre las mujeres desde una posición política de género. Por eso, la sororidad emerge como una alternativa a la política que confronta las violaciones de DDHH como responsabilidad de los Estados y que impide a las mujeres la identificación positiva de género, el reconocimiento, la agregación en sintonía y la alianza (Lagarde y de los Ríos, 2009).

El pacto de las mujeres transexuales en Chile y Latinoamérica conlleva una fuerte relación con la necesidad de construir una alianza entre ellas desde una posición política de género. Asimismo, trabajando fuertemente con el Sindicato chileno de Trabajadoras Sexuales Trans "Amanda Jofré", se ha evidenciado la expresión máxima de sororidad entre ellas, donde, por ejemplo, el soporte que ofrece la organización hacia las "compañeras nuevas que vienen llegando" se ha transformado en el arma más importante del colectivo, entregando información oportuna, necesaria y vital, en especial a las migrantes transexuales que llegan a Chile en búsqueda de nuevas oportunidades, movidas por diversas motivaciones.

Finalmente, se podría conjeturar que entre ellas son cómplices; inducen a las "nuevas" al mercado sexual, sororamente les enseñan cuánto cobrar por los servicios, les orientan en qué hacer en caso de emergencias, cómo utilizar la red de salud pública, las advierten de no dejarse pasar a llevar por la policía uniformada, y así entre un amplio arsenal de estrategias de supervivencia y compañerismo, están ellas pactando sus vidas, haciendo su trato.

Ellas saben que son invisibles frente al delito de la trata de personas, saben que no son reconocidas, saben quiénes operan estas crueles redes de compra-venta de seres humanos; ellas mismas han sido víctimas y supervivientes a la vez, ellas ponen en alerta a las menos experimentadas y se encargan de dar sepultura digna a las que han "caído en batalla". 
Ellas mismas han aprendido un nuevo estilo de conocimiento y destrezas, con los costes de desaprender la historia patriarcal que han heredado; ella mismas se han construido, desde la calle, desde la violencia, desde la segregación, y aun así, en esos paupérrimos escenarios, han sido reconocidas por sus pares y se han cuidado unas a otras.

Cuando hablo de "sororidad" hablo de ellas, de sus historias de calle, de sus actos dignos de humanidad, de su asistencia sanitaria artesanal, pues los sistemas de salud no las reconocen con sus demandas particulares; hablo de ellas, de sus códigos y expresiones, de sus convocatorias a la solidaridad con su propio género, de la solidaridad entre ellas mismas, de la fidelidad de un beso reservado siempre para el amor o amores de su vida, el resto es parte del trabajo.

\section{Bibliografía}

Aldunate, L. B. (1981). Constitución política de la República de Chile. Concordancias, Anotaciones y Fuentes, 5. Recuperado de http://scholar.google.com/scholar?hl=en\&btnG=Search\& $\mathrm{q}=$ intitle:Constitución+política+de+la+república+de+ch ile\# 1

Bales, K. (1999). "Disposable People" New Slavery in the Global Economy. California: University of California Press.

Datta, M. N. \& Bales, K. (2013). Slavery is Bad for Business: Analyzing the Impact of Slavery on National Economies. Brown Journal of World Affairs, 19(2), 205-223.

Delgado-Rodríguez, M. (2014). La salud de los inmigrantes en España Rev. Esp. Salud Pública, 88(6), 671-674.

Departamento de Crimen Organizado, División de Estudios, S. del I. (2012). Ministerio del Interior y Seguridad Pública. Santiago de Chile.

Espinosa, Y. (2012). La política sexual radical autónoma, sus debates internos y su crítica a la ideología de la diversidad sexual. En: Pensando los feminismos en Bolivia (pp. 113-126). Conexión Fondo de Emancipación, Serie Foros 2.

IOM/CHILE. (2008). Investigación sobre Trata de Personas en Chile Resumen Ejecutivo. Santiago de Chile. Recuperado de https:// es.scribd.com/document/128679744/Investigacion-sobre-trata-de-personas-en-Chile 
Kearney, M. (2008). Lo local y lo global: la antropología de la globalización y el transnacionalismo. En Hiernaux y Zarate (eds.), Espacios y transnacionalismo (pp. 51-88.). México: UAM Iztapalapa.

Lagarde y de los Ríos, M. (2009). Pacto entre mujeres: sororidad. Aportes para el Estado y la Administración Gubernamental, 25, 123-135. Recuperado de http://www.asociacionag.org.ar/ revista-aportes/edicion-25/

Norma, T., Medidas, E. y La, C. (2013). Biblioteca del Congreso Nacional de Chile - www.leychile.cl - documento generado el 04-jun-2013.

Santander, E., Sigrid, C., Primera, D. y Barrera, A. (2009). Normas de manejo y tratamiento de las Infecciones de Transmisión Sexual (ITS). Primera parte Sexually Transmitted Infections: Guidelines for their diagnosis and treatment. First Part. Rev Chil Infect, 26(2), 174-190. DOI: http://doi. org/10.4067/S0716-10182009000200012

UNODC. (2014). Global Report on Trafficking in Persons. New York. Recuperado de https://www.unodc.org/documents/dataand-analysis/glotip/GLOTIP_2014_full_report.pdf

Viveros Vigoya, M. (2016). La interseccionalidad: una aproximación situada a la dominación. Debate Feminista, 52, 1-17. DOI: http://doi.org/10.1016/j.df.2016.09.005

Zaldúa, G., Longo, R. y Sopransi. L. M. (2007). Exigibilidad de Derechos de personas en situación de prostitución. CABA, 6-8. 